\title{
Binocular information summation and the serial processing model ${ }^{1}$
}

An experiment was performed to ascertain whether a particular member of the class of sequential processing models (Estes \& Taylor, 1964; Townsend, 1966) should be further developed in terms of the contributions of information from the separate eyes. Using the detection paradigm (Estes \& Taylor, 1964), nine Ss were each run 576 trials under each of three viewing conditions: $(a)$ monocular left, (b) monocular right, and (c) binocular, after four days of practice and calibration. The serial processing model was used to make predictions for three possible cases of binocular information summation: (a) complete independence of the monocular channels, (b) partial independence of the monocular channels and (c) complete dependence in the monocular channels. Complete dependence provided the best fit to the data with a possible stress on use of a "best" eye, but a marginal level of significance was obtained between a simple average of monocular performance and binocular performance with transformed scores. Thus, although there seems to be little or no information summation in terms of the present model of multi-symbol perception, follow-up experiments were suggested to further delineate monocular-binocular relationships in the detection paradigm.

The visual span of apprehension has recently received new attention in the form of experiments that reduce the effects of immediate memory limitations on the estimated number of symbols apprehended (e.g., Sperling, 1960; Averbach \& Coriell, 1961; Estes \& Taylor, 1964). In particular, the basic Estes and Taylor (1964) method requires that $S$ recognize or detect which one of two predesignated signal symbols has been embedded in an array of noise symbols; we will refer to this method as the detection paradigm.

As in other experimental contexts, the question may be raised as to what extent the systems associated with the separate eyes function as independent information processors (i.e., to what extent the information from the separate eyes is additive). Broadbent's (1958) theory of the auditory system supposes independent short-term storage locations for the two ears and a subsequent selective filter and central processor; Sampson and his coworkers (Sampson \& Spong, 1961 a; Sampson \& Spong, 1961 b; Sampson, 1964) have attempted to test this model for immediate memory with different visual input to the two eyes. He tentatively concludes that Broadbent's theory of immediate memory "is a special case which, though not typical, is applicable to vision" (Sampson, 1964). If we apply this model to the case where the input to the two eyes is identical, then we should predict that in the Estes and Taylor detection paradigm $S$ will be able to store input from one eye while processing that from the other, then process any remaining information from the storage area. The present experiment is designed to test for this and other possibilities within the Estes detection paradigm by the use of three viewing conditions: (a) monocular left, (b) monocular right, and (c) binocular.

Several characteristics of choice behavior in the detection paradigm have been analyzed under varied stimulus conditions (Estes \& Taylor, 1964; Estes \& Wessel, 1966) and with varied presentation probabilities (Townsend, 1966). A class of models that is able to predict some of these characteristics when applied to this paradigm assumes that on each trial $S$ acquires a sample of symbols from the display that he then processes in a sequential fashion (Estes \& Taylor, 1964; Townsend, 1966). In the event that the signal letter is not contained in S's sample and/or the sample becomes unavailable to $S$ (i.e., falls below threshold before he processes the signal letter), $S$ is assumed to make his response by guessing. In particular, the Estes and Taylor serial processing model yields the following prediction for overall probability correct in the monocular viewing condition independent of response bias:

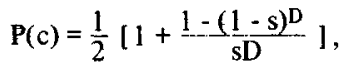

where $s$ is the probability that the sample of letters becomes available to $S$ at the end of each time interval $\Delta t(\Delta t$ is a constant representing the time required to process a single letter) following the offset of the stimulus display. The symbol $D$ stands for the D - 1 noise letters and the 1 signal letter present in the stimulus display.

This model was employed to make predictions for three underlying hypotheses concerning the summation of information from the separate channels:

(a) Complete independence of the separate eye channels. This could follow from two independent processors, one for each eye, or from perfect storage of one eye's input while the central processor works on input from the other eye (as in a strong interpretation of Broadbent's model [1958]). The predicted probability correct for this hypothesis is

$$
\begin{aligned}
P(c)= & \frac{1}{2}\left[1+\frac{1-\left(1-s_{1}\right)^{D}}{s_{1}{ }^{D}}+\frac{1-\left(1-s_{\mathrm{T}}\right)^{D}}{s_{\mathrm{r}}^{D}}\right. \\
& \left.-\frac{1-\left(1-s_{1}\right)^{D}-\left(1-s_{\mathrm{T}}\right)^{D}+\left(1-s_{\mathrm{t}}\right)^{D}\left(1-s_{\mathrm{r}}\right)^{D}}{s_{1} \mathrm{~s}_{\mathrm{T}} \mathrm{D}^{2}}\right],
\end{aligned}
$$

where $1=$ monocular left, and $r=$ monocular right.

(b) Partial independence of the separate eye channels. If the central processor works on one channel while the information continues to decay independently in the other (as in a weaker interpretation of Broadbent's model), or if the letters of the sample are "superimposed" but decay continues independently in the two channels, the processor continuing to work until the last (second) channel falls below threshold, the predicted probability correct would fall between that for complete independence and that for complete dependence:

$P(c)=\frac{1}{2}\left[1+\frac{1-\left(1-s_{1}\right)^{D}}{s_{1}^{D}}+\frac{1-\left(1-s_{\mathrm{r}}\right)^{D}}{s_{T}^{D}}-\frac{1-\left(1-s_{1}\right)^{D}\left(1-s_{\tau}\right)^{D}}{\left.11-\left(1-s_{1}\right)\left(1-s_{\tau}\right)\right] D}\right]$

(c) Complete dependence in the separate eye channels. This hypothesis is generated by assuming that either the information from the two channels to a central processor is combined and is characterized by a single average deeay parameter or that on some proportion of the trials $S$ processes information from the left eye alone and on the remaining trials from the right eye alone (no Broadbent storage of single channel information takes place). In contrast to possibilities mentioned for (a) and (b), these two possibilities are theoretically distinct for the serial processing model, but they make indistinguishable predictions for the present experiment when we assume equal weight on use of the two channels. Probability of a correct response is then given by

$$
P(c)=\frac{1}{2}\left[P_{1}(c)+P_{r}(c)\right],
$$

where $P_{1}(c)$ is the observed probability correct for the left eye and $P_{r}(c)$ is the observed probability correct for the right eye.

In an attempt to preclude effects of binocular energy summation in favor of what we refer to as information summation, as given in various degrees by the hypotheses above, pilot conditions were run and energy levels selected which insured essentially perfect perception of one or two letters, both for binocular and monocular conditions. 


\section{METHOD}

Apparatus

Stin.ulus presentation was effected by means of a tachistoscope modified to allow for monocular and binocular viewing conditions. Exposure was provided by a cold-cathode fluorescent tube that provided uniform illumination of the stimulus display, which was positioned 20-1/2 in. from the eyes of Ss. Between exposures, the stimulus field was dark except for a centered fixation light, approximately 1/64 in. in diameter. During exposures, the luminance of the stimulus field was $7-1 / 2 \mathrm{ft}-\mathrm{L}$ and the ambient light level was essentially zero as measured by a Macbeth illuminometer. The viewing hood was so constructed that a small black plate could be fitted in the hood to exclude vision in the right or left eye, without pressing against S's eye.

\section{Stimulus materials}

The stimulus displays were arrays of 16 consonants (Fig. 1) 15 of which were always "noise" symbols and one was always a "signal" symbol ( $R$ or $H)$. For each array containing an $R$ there was a corresponding $\mathrm{H}$ array possessing the same arrangement of noise letters. Each signal symbol appeared exactly once in each of the 16 possible symbol positions; thus, there were 32 stimulus displays in all. These stimulus displays were typed in the IBM Executive Directory style, .14 type, code 66 , pk, type style. The array of letters subtended an angle of $2 \mathrm{deg} 55 \mathrm{~min}$ and an individual letter $40 \mathrm{~min}$ at $\mathrm{S}$ 's eye.

\section{Subjects and Procedure}

Nine Ss drawn from the Stanford University community were paid for their participation in the experiment. Three were people used in a previous pilot study and six were naive Ss. Before beginning the experiment each $S$ was administered a simple acuity test and the ring test for eye dominance. ${ }^{3}$ All Ss except one possessed 20/20 acuity in both eyes after correction (glasses could be worn with the apparatus); the exception had 20/20 acuity in one eye and $20 / 30$ in the other. Right eye dominance was observed in five of the nine Ss, left eye dominance in three Ss, and one $S$ possessed no clearly dominant eye. The acuity and eye dominance examinations were repeated after the experiment with identical findings.

The S's task was to use two response buttons to indicate whether an $R$ or an $H$ had been embedded in the display. Following the symbol response $S$ was requested to give a rating of 1 to 6 to indicate his degree of confidence in his choice, ranging from absolute certainty (1) to guessing at random (6). Correct feedback was always supplied $\mathbf{S}$.

Four days were taken with each $S$ to acquaint him with the apparatus and to establish a stimulus duration under which he averaged about $75 \%$ correct over the three viewing conditions (monocular left, monocular right, and binocular). The final stimulus display times for the nine Ss ranged from a minimum of 4 msec to a maximum of $150 \mathrm{msec}$. During these four days the six

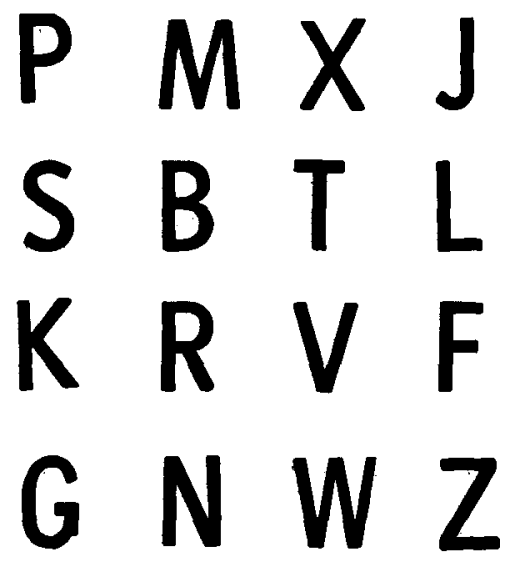

Fig. 1. A typical stimulus array, containing the $R$ signal symbol. naive Ss received 275 trials in each of the three viewing conditions with the order of the viewing conditions counterbalanced across Ss. The three experienced Ss were treated exactly the same except that due to an experimental error, 288 practice trials were run under each viewing condition.

Following the practice phase an experimental phase began that lasted six sessions. Each session consisted of 288 trials partitioned into three basic sequences. A basic sequence was composed of three blocks of 32 trials each, one block being run under each of the three viewing conditions. Each block of 32 trials was generated by shuffling the deck of 32 possible stimulus arrays; thus, each block of 32 trials contained $16 \mathrm{R}$-trials and $16 \mathrm{H}$-trials presented in a random fashion. Each $S$ received every permutation of the three viewing conditions over the six sessions and the order of the various permutations was randomized across Ss.

\section{RESULTS AND DISCUSSION}

Figure 2 shows the average proportion (estimated probability) correct under each viewing condition for each of the nine Ss. Confidence bands for $\pm 2 \sigma_{M}$ are shown with the points. Pictured with the binocular points are the predictions obtained from the three hypotheses after estimating $s_{1}$ and $s_{r}$ for each $S$. For six of the nine Ss, the complete dependence prediction is superior to the other predictions although for two of these, the prediction does not lie within the confidence limits of the observed binocular point. A fourth hypothesis, really a limiting case of complete dependence, assumes that $S$ relies heavily or completely on his best eye. Since it turned out that S's dominant eye, as defined by the ring test, bore no discernible relation to his performance, there was no a priori way to define "best." However, it is interesting that proportion correct for the observed best eye lies within the confidence bounds of the binocular point in seven of the nine cases and one of the exceptions satisfies the complete dependence prediction perfectly.

As indicated by a comparison of Fig. 2 with Fig. 3, confidence ratings seemed to be related to proportion correct across conditions for some Ss but not for others; overall the Ss do not appear substantially more confident under the binocular condition. That this finding did not follow from an inability to relate their likelihood of being correct to their ratings is shown by the monotonic relationship depicted in Fig. 4.

Although the complete dependence hypothesis as expressed in an averaging rule seems to provide the best approximation to the data, because the binocular proportion correct was greater than either monocular point for five Ss, further statistical analyses were performed. First, a two-way analysis of variance (S by viewing condition) on proportion correct, $\mathbf{P}(\mathrm{c})$, indicated a nonsignificant viewing condition effect, $F=3.55$, $\mathrm{df}=2 / 16, \mathrm{p}>.05$. However, when the same analysis of variance model was applied to arcsin $\sqrt{\mathrm{P}(\mathrm{c})}$, and following Myers' (1966, pp. 160-162) suggestion for dealing with possible heterogeneity of covariances, significance was found between the .01 and .05 levels $(F=3.90$, $\mathrm{df}=2 / 16$, $.05>p>.01)$. A subsequent one-tailed test between the average monocular arcsin $\sqrt{\mathrm{P}(\mathrm{c})}$ and the binocular $\arcsin \sqrt{\mathrm{P}(\mathrm{c})}$ revealed significance at the .05 level $(t=1.98, \mathrm{df}=8, .05>\mathrm{p})$. Since an increased probability of incorrectly rejecting the null hypothesis is usually expected with data violating analysis of variance assumptions (see, e.g., Lindquist's discussion of Norton's dissertation, 1953, pp. 78-90), there may be a "real" but small difference present, at least between a simple average of the monocular conditions and the binocular condition.

In summary, a simple average of proportion correct for the monocular conditions (complete dependence) was shown to predict binocular proportion correct much better than the partial or complete independence hypotheses, under the corresponding interpretations of the serial processing model. ${ }^{4}$ In particular, any moderate or strong interpretation of the Broadbent assumptions of separate storage for the two monocular channels seems to be negated for the detection paradigm when binocular input is to overlapping visual fields. Since the nature of the detection paradigm minimizes the importance of (post-processing) immediate memory, the effects of monocular storage antecedent 


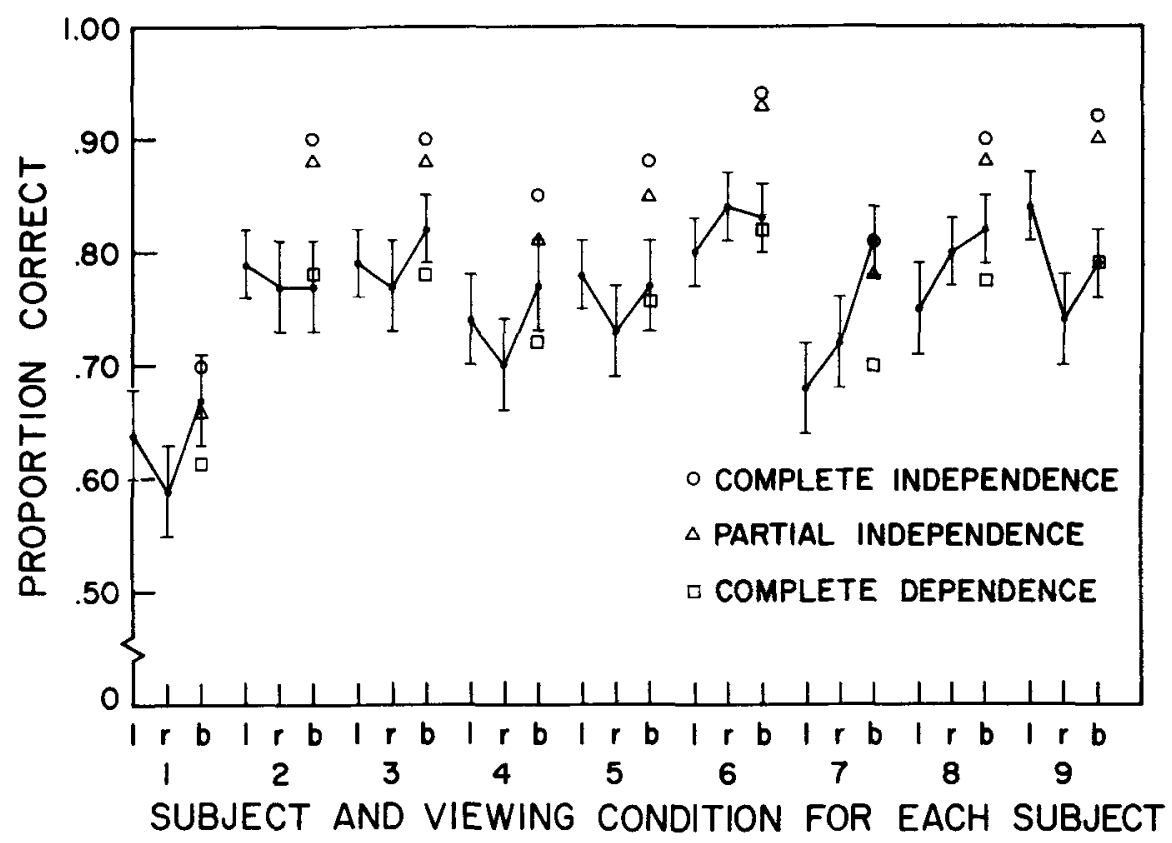

Fig. 2. Relation between viewing condition and proportion correct for each $\mathbf{S}$ and each viewing condition, and three predictions from the serial processing model. $(1=$ monocular left, $r=$ monocular right, $b=$ binocular; confidence bands represent $\pm 2 \sigma_{M}$.)

to central processing might have been expected to be especially salient. In this context, it should be noted that when preprocessing storage time has been investigated directly (Sperling, 1960; Averbach \& Coriell, 1961) it usually is found to be of relatively small magnitude: about $250 \mathrm{msec}$. This kind of storage may be of peripheral nature (e.g., afterimages) and especially sensitive to pattern masking effects; these effects would limit the usefulness of this kind of storage in sequential-presentation immediate memory tasks. However, it should be interesting to carry out the present experiment in the case where binocular input is to separate visual fields in the two eyes.

Since statistical significance was obtained between a simple average of monocular arcsin $\sqrt{\mathrm{P}(\mathrm{c})}$ and binocular arcsin $\sqrt{\mathrm{P}(\mathrm{c})}$, though not for the untransformed scores, judgment should perhaps be reserved on the question of whether there is any binocular interaction present. If there is a real difference, it might follow from slight information summation, a failure to exclude all energy summation effects, or simply a slight bias in favor of binocularity associated with its customary use and comfort in normal vision. Given the lack of a consistent binocular superiority across $S s$ and the apparent sizable contribution to statistical significance by one $\mathbf{S}$ (S No. 7), the most attractive explanation for the present results is perhaps a more general averaging rule with a favoring of the better eye. It is clear that the partial and complete independence hypotheses, as incorporated in the serial processing model, are grossly inadequate for the present data.

It is not surprising, perhaps, that no large difference was found between the monocular and binocular conditions, given the convergence of corresponding retinal fibers in the two eyes to the same point in the visual cortex (Hubel \& Wiesel, 1962). However, it might have been similarly predicted from relative independence of photochemical decay and recovery in the two retinae and/or the possibility of peripheral coding that the partial independence model would apply.

A follow-up study to further assess the monocular contributions to binocular vision might include a phase wherein the best eye is determined statistically under conditions identical to those in the experiment proper, as well as a condition in which twice the luminous flux is put into one eye as a control for energy summation. Another condition of interest involves monocular viewing with one eye for half the binocular stimulus duration and monocular viewing with the other eye for the second half of the

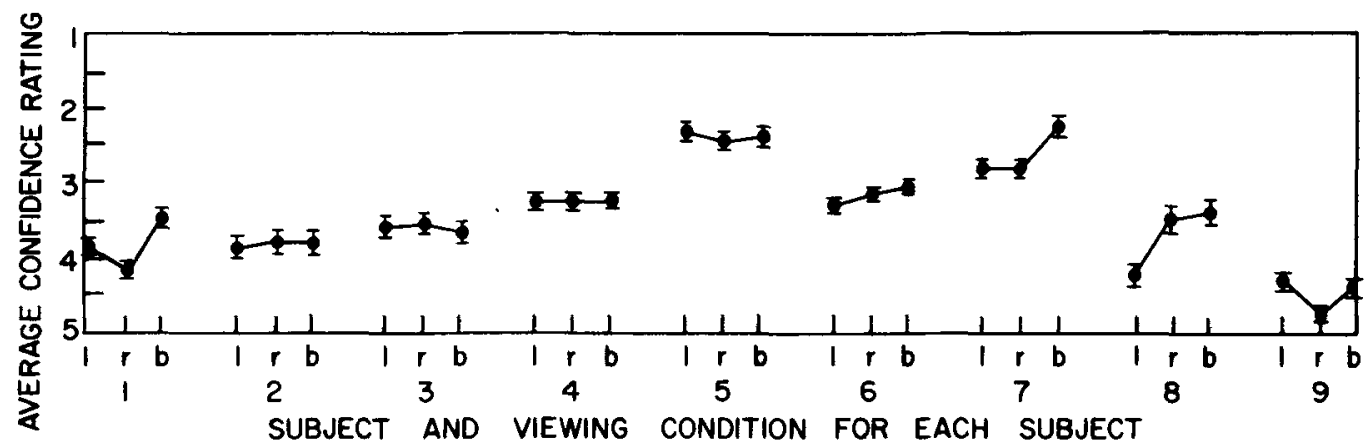

Fig. 3. Average confidence rating for each $S$ and each viewing condition. $(I=$ monocular left, $r=$ monocular right, $b=$ binocular; confidence bands represent $\pm 2 \sigma_{M}$.) 


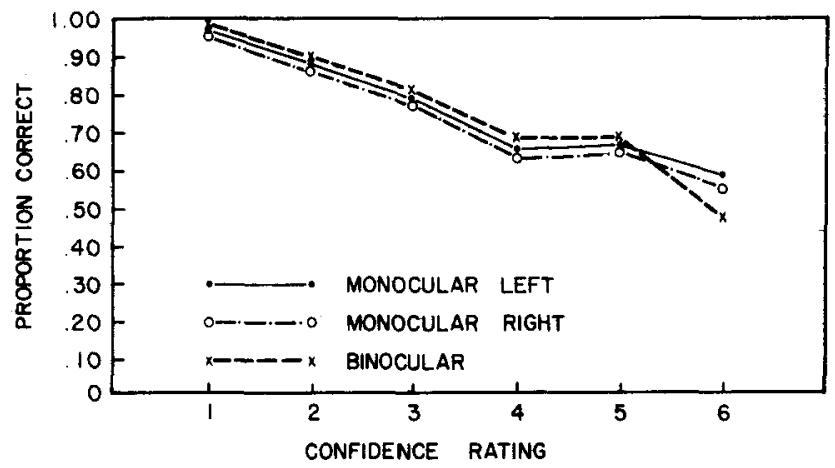

Fig. 4. Proportion correct based on viewing condition and confidence rating, averaged over Ss.

stimulus duration, with perhaps an inter-eye interval of no stimulation to insure no energy summation. This condition would provide a partial physical realization of the complete independence hypothesis for binocular viewing.

Assuming the results of the above experiment again support some averaging rule, it would be of value to attempt to determine whether the output of each eye is being averaged continuously (as might be expected from a fusion theory of binocular vision, e.g., Fleischer, 1937), or whether there is an alternation between eyes in binocular viewing (as suggested by Neuhaus, 1936). The first hypothesis is more compatible with Hubel and Wiesel's (1962) finding that binocular enhancement occurs with recording from single cells in the visual cortex. The second seems to be in agreement with the majority of binocular rivalry data (see e.g., Asher, 1966), but is presently inadequate to fully explain the phenomena of binocular color mixture. One tentative way of investigating these two possibilities within the context of the detection paradigm would be to obtain monocular and binocular performance curves as a function of stimulus duration, perhaps with compensating monocular energy input to exclude energy summation effects. The alternation theory predicts (assuming that switching time from one eye to the other is not insignificant relative to our shorter tachistoscopic exposure durations) that at some critical stimulus duration we should be confronted with the surprising result of binocular inferiority relative to monocular performance. The result of no difference would offer some support for a fusion theory or that version of alternation theory that assumes alternate suppression of various corresponding areas, rather than alternation from one eye to the other (see, e.g., Walls, 1942 , p. 324; Verhoeff, 1935). If only a few areas alternated at any one time, no appreciable difference between conditions would be expected in the above experiment. However, it seems difficult to devise strong tests for these theories without further specification of the hypothetical structure and/or possible underlying mechanisms involved in binocular vision.

\section{REFERENCES}

ASHER, H. A. Experiments in seeing. New York: Fawcett World Library, 1966.

AVERBACH, E., \& CORIELL, A. S. Short-term memory in vision. Bell System Tech. J., 1961. Pp. 309-328.

BROADBENT, D. E. Perception and communication. London: Pergamon Press, 1958.

ESTES, W. K., \& TAYLOR, H. A. A detection method and probabilistic models for assessing information processing from brief visual displays. Proc. NAS, 1964, 52, No. 2, 446-454.

ESTES, W. K., \& TAYLOR, H. A. Visual detection in relation to display size and redundancy of critical elements. Percept. \& Psychophys., 1966, 1, 9-16.

ESTES, W. K., \& WESSEL, D. L. Reaction time in relation to display size and correctness of response in forced-choice visual signal detection. Percept. \& Psychophys., 1966, 1, 369-373.

FLEISCHER, E. Die Granzlinienerregungen beim Sehen mit beiden Augen. Ztschr. f. Psychol. u. Physiol. d. Sinnesorg., Abt. 1, 1937, 141, 283-342. Cited by K. N. Ogle, Researches in binocular vision. New York: Hafner, 1950.

HUBEL, D. H., \& WIESEL, T. N. Receptor fields, binocular interaction and functional architecture in the cat's visual cortex. J. Physiol, 1962,160, 106-154.

LINDQUIST, E. F. Design and analysis of experiments in psychology and education. Boston: Houghton Mifflin, 1953.

MYERS, J. L. Fundamentals of experimental design. Boston: Allyn and Bacon, 1966.

NEUHAUS, W. Experimentelle Untersuchung über das Sehen mit beiden Augen. Ztschr. f. Psychol. u. Physiol d. Sinnesorg., Abt. I, 1936, 137, $87-108$. Cited by K. N. Ogle, Researches in binocular vision. New York: Hafner, 1950.

SAMPSON, H. Immediate memory and simultaneous visual stimulation. Quart. J. exp. Psychol., 1964, 16, 1-10.

SAMPSON, H., \& SPONG, P. Binocular fixation and immediate memory. Brit. J. Psychol, 1961 a, 52, 239-248.

SAMPSON, H., \& SPONG, P. Handedness, eye-dominance and immediate memory. Quart. J. exp. Psychol., 1961b, 13, 173-180.

SPERLING, G. The information available in brief, visual presentations. Psychol. Monogr., 1960, 74, No. 11 (Whole No. 498).

TOWNSEND, J. T: Choice behavior in a cued-recognition task. Tech. Rep. No. 103, Psychol. Ser., Inst. for Mathematical Studies in the Social Sciences, Stanford University, 1966.

VERHOEFF, F. H. A new theory of binocular vision. Arch. Ophth, n.s., $1935,13,151-175$.

WALLS, G. L. The vertebrate eye. Bloomfield Hills: Cranbrook Institute of Science, 1942.

\section{NOTES}

1. This study was carried out under the sponsorship of Professor Richard C. Atkinson while the author was an NDEA predoctoral fellow at the Institute for Mathematical Studies in the Social Sciences at Stanford University. The author is also indebted to Professor William J. McGill (University of California, San Diego) and Professor Hiroshi Ono (University of Hawaii) for their valuable comments.

2. Address: Department of Psychology, Purdue University, Lafayette, Indiana 47907.

3. The ring test is a simple method for determining eye preference. The $S$ holds a small ring about $1 \mathrm{ft}$ from his face without looking through it. At a signal from $\mathrm{E}, \mathrm{S}$ must look through it and fixate on a more distant object designated by $\mathrm{E}$. Since the ring is too close for binocular fixation through the ring, $\mathrm{S}$ must move the ring in front of one of his eyes. The assumption is that the eye most often chosen is dominant.

4. It should be noted that for the present experiment, the complete independence and complete dependence predictions follow from any simple threshold model of the form $P(c)=1 / 2[1+P]$, when $P$ represents the probability of obtaining a correct response through sensory (as distinguished from guessing) means.

(Accepted for publication April 18, 1968.) 